\title{
GCE AND SOLAR S-PROCESS ABUNDANCES: DEPENDENCE ON THE ${ }^{13} \mathrm{C}$-POCKET STRUCTURE
}

\section{Bisterzo, S.*}

INAF - Astrophysical Observatory Turin, Turin, Italy

University of Turin, Physics Department, Turin, Italy

E-mail: bisterzo@to.infn.it, sarabisterzo@gmail.com

Travaglio, C.

INAF - Astrophysical Observatory Turin, Turin, Italy

B2FH Association, Turin, Italy

\section{Gallino, R.}

University of Turin, Physics Department, Turin, Italy

Wiescher, M.

Joint Institute for Nuclear Astrophysics (JINA), Physics Department, University of Notre Dame,

Notre Dame, IN

Käppeler, F.

Karlsruhe Institute of Technology (KIT), Institut fuer Kernphysik, Karlsruhe, Germany

\begin{abstract}
We investigate the impact of asymptotic giant branch (AGB) stellar yields computed with different internal structures of the ${ }^{13} \mathrm{C}$ pocket (which may influence the efficiency of the major neutron source of the $s$ process) on Galactic Chemical Evolution (GCE) $s$-process predictions at the epoch of the solar system formation. The aim of this work is to implement the analysis given by [6]. We extend the analysis at different metallicities, and we compare the GCE results with updated spectroscopic observations of selected neutron capture elements.
\end{abstract}

XIII Nuclei in the Cosmos,

7-11 July, 2014

Debrecen, Hungary

\footnotetext{
*Speaker.
} 


\section{GCE SOLAR s-PROCESS PREDICTIONS}

We study the solar abundances of $s$ isotopes $(A>90)$ in the framework of the chemical evolution of the Galaxy. We focus our analysis on one of the most problematic issues of the $s$ process nucleosynthesis in low-mass AGB models: the formation of the ${ }^{13} \mathrm{C}$ pocket, where the major neutron source (the ${ }^{13} \mathrm{C}(\alpha, \mathrm{n}){ }^{16} \mathrm{O}$ reaction) burns radiatively in the interpulse phase. An unknown mechanism allows partial mixing of a few protons from the bottom of the convective H-rich envelope into the top layers of the radiative He- and C-rich intershell at the quenching of a Third Dredge Up (TDU) episode. When the star contracts, the $\mathrm{H}$ shell reignites and protons in the intershell are captured by the abundant ${ }^{12} \mathrm{C}$ nuclei to form primary ${ }^{13} \mathrm{C}$ according to the profile of diffused hydrogen. In the outer layers, for $\mathrm{p} /{ }^{12} \mathrm{C} \geq 1$, a region of primary ${ }^{14} \mathrm{~N}$ may form by further proton captures on ${ }^{13} \mathrm{C}$. Subsequently, the temperature in the ${ }^{12} \mathrm{C}$ pocket increases to $\sim 1 \times 10^{8} \mathrm{~K}$, neutrons are released radiatively within the pocket at quite low neutron densities and produce the $\mathrm{s}$ isotopes. The internal structure of the ${ }^{13} \mathrm{C}$ pocket may depend on the characteristic of the star (e.g., AGB initial mass and initial chemical composition), and on the interplay between various physical mechanisms that may compete (e.g., overshooting, rotation, magnetic fields, gravity waves; $[16,22,11$, $36,38,31,8,42])$.

For a given metallicity, spectroscopic observations in chemically peculiar $s$-rich stars show a scatter (see e.g., MS, S, C(N), Ba stars, post-AGB stars, planetary nebulae, CEMP-s). This spread has been recognized since the first studies by [7, 1] (see also the recent review [20, 19]).

Rotation may be regarded as a possible explanation of the observed scatter in the $s$-process distribution (see e.g., [31] and references therein). Studies on magnetic fields may provide additional information on the ${ }^{13} \mathrm{C}$-pocket structure [30].

The solar $s$-process abundances must account for the complex chemical evolution of the Galaxy, which includes AGB yields of various masses, metallicities, as well as the observed s-process scatter. For this reason we adopt a range of ${ }^{13} \mathrm{C}$-pocket strengths in our AGB models (the adopted AGB yields are presented by $[5,6])$. The range of ${ }^{13} \mathrm{C}$-pockets is constrained by comparing spectroscopic abundances of $s$-rich stars at each metallicity with AGB predictions (see, e.g., [20], their Figure 12).

The solar $s$-process abundances are reproduced by using the GCE code described by $[39,41]$ (we refer to these papers for further details on the GCE model). Starting from the computed range of ${ }^{13} \mathrm{C}$-pockets, we have calibrated the ${ }^{13} \mathrm{C}$-pocket strengths to be included in the GCE analysis in order to reproduce $100 \%$ of solar ${ }^{150} \mathrm{Sm}$, which is taken as reference isotope for the whole $s$ process distribution (see, e.g., Fig. 4 of [6]). In the framework of GCE, the weighted average among the ${ }^{13} \mathrm{C}$-pocket strengths is only constrained by the correct reproduction of the solar s-only ${ }^{150} \mathrm{Sm}$. However, a proper GCE weighted average among the ${ }^{13} \mathrm{C}$-pocket strengths may depend on the choice of the internal structure of the ${ }^{13} \mathrm{C}$ pocket.

We explore the impact of different shapes and sizes of the adopted ${ }^{13} \mathrm{C}$ pocket on the solar $s$ distribution. From the tests provided in [6], we have found that, once we assume a different weighted average of ${ }^{13} \mathrm{C}$ pockets, the GCE solar s predictions do not depend on the pocket structure.

The aim of this work is to implement the analysis given by [6]. On the base of the recent studies on the ${ }^{13} \mathrm{C}$-pocket issue, we are encouraged to extend the study to specific additional tests. 


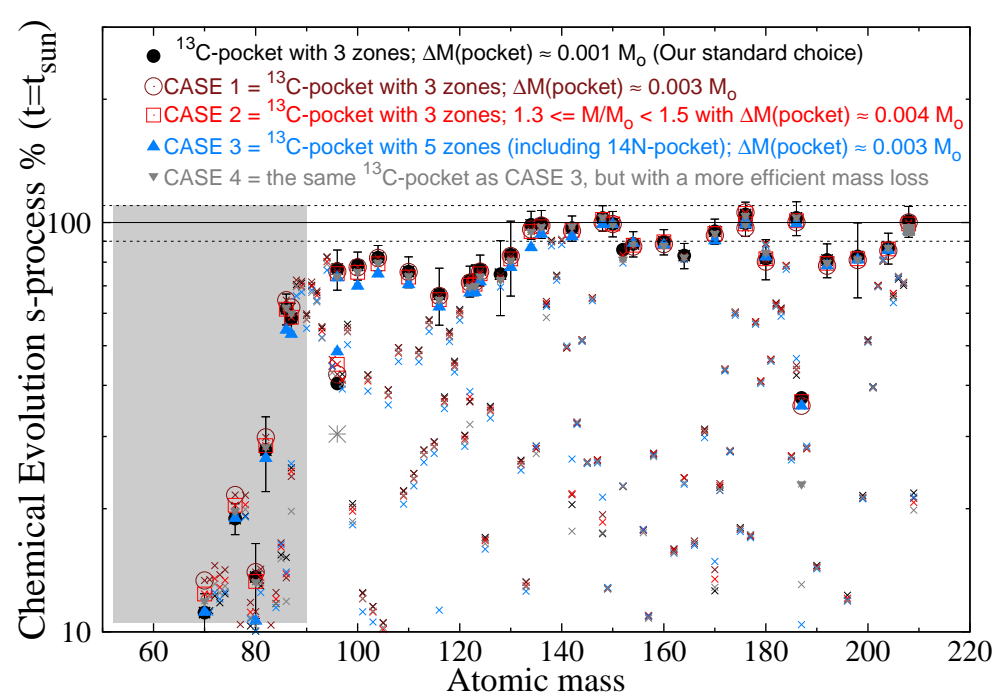

Figure 1. Effect of the ${ }^{13} \mathrm{C}$-pocket uncertainties on solar $s$-process predictions computed with the GCE model. The $s$-only isotopes are diplayed with filled circles. Isotopes with $A<90$ (gray zone, e.g., ${ }^{86,87} \mathrm{Sr}$ ) receive an additional contribution from the weak $s$-process in massive stars [32].

We present the most significant ones:

CASE 1 - in low-mass AGB models of $M=1.3$ to $3 M_{\odot}$, the mass of the ${ }^{13} \mathrm{C}$ pocket is increased by a factor of three with respect to our standard ${ }^{13} \mathrm{C}$-pocket mass (see, e.g., previous investigations on CEMP-s stars by [25]), but keeping the ${ }^{13} \mathrm{C}$ and ${ }^{14} \mathrm{~N}$ mass fractions unchanged;

CASE 2 - in low-mass AGB models of $M=1.3$ to $1.5 M_{\odot}$ models, the mass of the ${ }^{13} \mathrm{C}$-pocket is assumed a factor of four larger than in our standard models (e.g, magnetic buoyancy might be at the origin of the mixing process that produces an extended ${ }^{13} \mathrm{C}$-pocket, as $[8,26]$ suggest to occur in $M<1.5 M_{\odot}$ models);

CASE 3 - we add two external zones in the ${ }^{13} \mathrm{C}$-pocket with $X\left({ }^{14} \mathrm{~N}\right)$ much higher than $X\left({ }^{13} \mathrm{C}\right)$ (which simulates the poison effect of a ${ }^{14} \mathrm{~N}$-pocket, which may form where protons are enough to complete the $\mathrm{CN}$ cycle through ${ }^{13} \mathrm{C}(\mathrm{p}, \gamma){ }^{14} \mathrm{~N}$, see, e.g., $\left.[13,10]\right)$;

CASE 4 - the same as CASE 3, but we assume that a more efficient mass loss reduces the number of thermal pulses with third dredge-up for $M=1.5,2$ and $3 M_{\odot}$ models (in agreement with updated opacities and a revised luminosity function of Galactic carbon stars taking into account more recent infrared observations of AGB stars [23, 10, 14]).

The internal structure of the ${ }^{13} \mathrm{C}$ pocket adopted in each test is given in Table 1 . The range of ${ }^{13} \mathrm{C}$-pocket strengths is derived by multiplying the abundances of ${ }^{13} \mathrm{C}$ and ${ }^{14} \mathrm{~N}$ from 0 to 2 times the values of each zone given in Table 1.

We display the results of the above tests in Fig. 1. The $s$-only isotopes in the atomic mass range between $A=96$ to 208 show variations smaller than $\sim 10 \%$.

Despite ${ }^{208} \mathrm{~Pb}$ is not an $s$-only isotope, its solar abundance provides a strong constraint in the framework of GCE. ${ }^{208} \mathrm{~Pb}$, the double-magic nucleus $(\mathrm{N}=126 ; \mathrm{Z}=82)$ located at the termination point of the s process, is characterized by a small neutron capture cross section $(0.376 \pm 0.040 \mathrm{mb})$ and is the most abundant $\mathrm{Pb}$ isotope in the solar system (58.8\% of solar $\mathrm{Pb}$ is composed by ${ }^{208} \mathrm{~Pb}$ iso- 
Table 1. Internal structure of the ${ }^{13} \mathrm{C}$-pockets adopted in the tests displayed in Fig. 1.

\begin{tabular}{|c|c|c|c|c|c|}
\hline & zone I & zone II & zone III & zone IV & zone V \\
\hline \multicolumn{6}{|c|}{ Three-zone model with $M$ (pocket) $=1.09 \mathrm{E}-3 M_{\odot}$} \\
\hline $\operatorname{Mass}\left(M_{\odot}\right)$ & $5.50 \mathrm{E}-4$ & $5.30 \mathrm{E}-4$ & $7.50 \mathrm{E}-6$ & - & - \\
\hline$X\left({ }^{13} \mathrm{C}\right)$ & $3.20 \mathrm{E}-3$ & $6.80 \mathrm{E}-3$ & $1.60 \mathrm{E}-2$ & - & - \\
\hline$X\left({ }^{14} \mathrm{~N}\right)$ & $1.07 \mathrm{E}-4$ & $2.08 \mathrm{E}-4$ & $2.08 \mathrm{E}-3$ & - & - \\
\hline \multicolumn{6}{|c|}{$C A S E$ 1: three-zone model with $M$ (pocket) $=3 \times 1.09 \mathrm{E}-3 M_{\odot}$} \\
\hline $\operatorname{Mass}\left(M_{\odot}\right)$ & $1.65 \mathrm{E}-3$ & $1.59 \mathrm{E}-3$ & $2.25 \mathrm{E}-5$ & - & - \\
\hline$X\left({ }^{13} \mathrm{C}\right)$ & $3.20 \mathrm{E}-3$ & $6.80 \mathrm{E}-3$ & $1.60 \mathrm{E}-2$ & - & - \\
\hline$X\left({ }^{14} \mathrm{~N}\right)$ & $1.07 \mathrm{E}-4$ & $2.08 \mathrm{E}-4$ & $2.08 \mathrm{E}-3$ & - & - \\
\hline \multicolumn{6}{|c|}{ CASE 2: $1.3 \leq M<1.5 M_{\odot}$ models with $M$ (pocket) $=4 \times 1.09 \mathrm{E}-3 M_{\odot}$} \\
\hline $\operatorname{Mass}\left(M_{\odot}\right)$ & $2.20 \mathrm{E}-3$ & $2.12 \mathrm{E}-3$ & $3.00 \mathrm{E}-5$ & _- & _- \\
\hline$X\left({ }^{13} \mathrm{C}\right)$ & $3.20 \mathrm{E}-3$ & $6.80 \mathrm{E}-3$ & $1.60 \mathrm{E}-2$ & - & - \\
\hline$X\left({ }^{14} \mathrm{~N}\right)$ & $1.07 \mathrm{E}-4$ & $2.08 \mathrm{E}-4$ & $2.08 \mathrm{E}-3$ & - & - \\
\hline \multicolumn{6}{|c|}{ CASE 3: five-zone model with external ${ }^{14} \mathrm{~N}$-rich zones } \\
\hline $\operatorname{Mass}\left(M_{\odot}\right)$ & $1.20 \mathrm{E}-3$ & $1.05 \mathrm{E}-3$ & $3.30 \mathrm{E}-4$ & $3.30 \mathrm{E}-4$ & $3.30 \mathrm{E}-4$ \\
\hline$X\left({ }^{13} \mathrm{C}\right)$ & $3.20 \mathrm{E}-3$ & $6.80 \mathrm{E}-3$ & $1.60 \mathrm{E}-2$ & $4.00 \mathrm{E}-2$ & $4.00 \mathrm{E}-2$ \\
\hline$X\left({ }^{14} \mathrm{~N}\right)$ & $1.07 \mathrm{E}-4$ & $2.08 \mathrm{E}-4$ & $2.08 \mathrm{E}-3$ & $8.00 \mathrm{E}-2$ & $1.49 \mathrm{E}-1$ \\
\hline
\end{tabular}

tope). Starting first studies by [12, 3], it was evident that about $50 \%$ of solar ${ }^{208} \mathrm{~Pb}$ is missing when low metallicity AGB models are not considered. GCE results by [40] demonstrated that low-mass AGB stars are the main producers of $\mathrm{Pb}$ in the Galaxy, with a complex dependence on metallicity and a maximum efficiency at $[\mathrm{Fe} / \mathrm{H}] \sim-1$. Specifically, they found that low-mass low-metallicity AGB stars explain the missing $50 \%$ of solar ${ }^{208} \mathrm{~Pb}$.

\section{GCE s- and r-process contributions versus $[\mathrm{Fe} / \mathrm{H}]$}

We compare the GCE predictions of $\mathrm{Y}, \mathrm{Ba}$, and $\mathrm{Pb}$ (representative of the three peaks of the $s$ process distribution), and $\mathrm{Eu}$ (among the $r$ process elements), with the most updated spectroscopic observations of $[\mathrm{Ba} / \mathrm{Fe}],[\mathrm{Eu} / \mathrm{Fe}],[\mathrm{Y} / \mathrm{Fe}],[\mathrm{Pb} / \mathrm{Fe}]$ and their ratios $[\mathrm{Ba} / \mathrm{Eu}],[\mathrm{Eu} / \mathrm{Y}],[\mathrm{Ba} / \mathrm{Y}]$ and $[\mathrm{Pb} / \mathrm{Eu}]$ versus $[\mathrm{Fe} / \mathrm{H}]$ (see Figure 2). Different lines correspond to GCE results obtained with different tests selected from Section 1: black line represents the standard three zones ${ }^{13} \mathrm{C}$-pocket, blue line is CASE 1 and green line is CASE 3. We also display the result of a flat ${ }^{13} \mathrm{C}$-pocket profile obtained by excluding the two external ${ }^{13} \mathrm{C}$-rich zones of the pocket (see [6]) with magenta line.

The $r$-rich stars with $[\mathrm{Eu} / \mathrm{Fe}] \geq 1$ at $[\mathrm{Fe} / \mathrm{H}]<-2([27,35])$ are peculiar objects extremely enhanced in $r$-process elements, likely born from a cloud polluted by SNe. The same objects have $[\mathrm{Ba}, \mathrm{Y}, \mathrm{Pb} / \mathrm{Fe}]$ higher than the average of field stars. They may provide information on the pure $r$-process contribution, but they do not represent the average chemical evolution of our Galaxy.

The GCE $r$ contribution is estimated with the residual method $N_{r}=N_{\odot}-N_{s}$. As discussed by [39], the $r$-process contribution is assumed to derive from SNII of 8-10 $M_{\odot}$. However, we do not exclude different hypotheses owing to the poor knowledge on the stellar mass range that contributes to the production of heavy r process elements (see e.g., [28, 21]). Indeed, by adopting different ranges of 


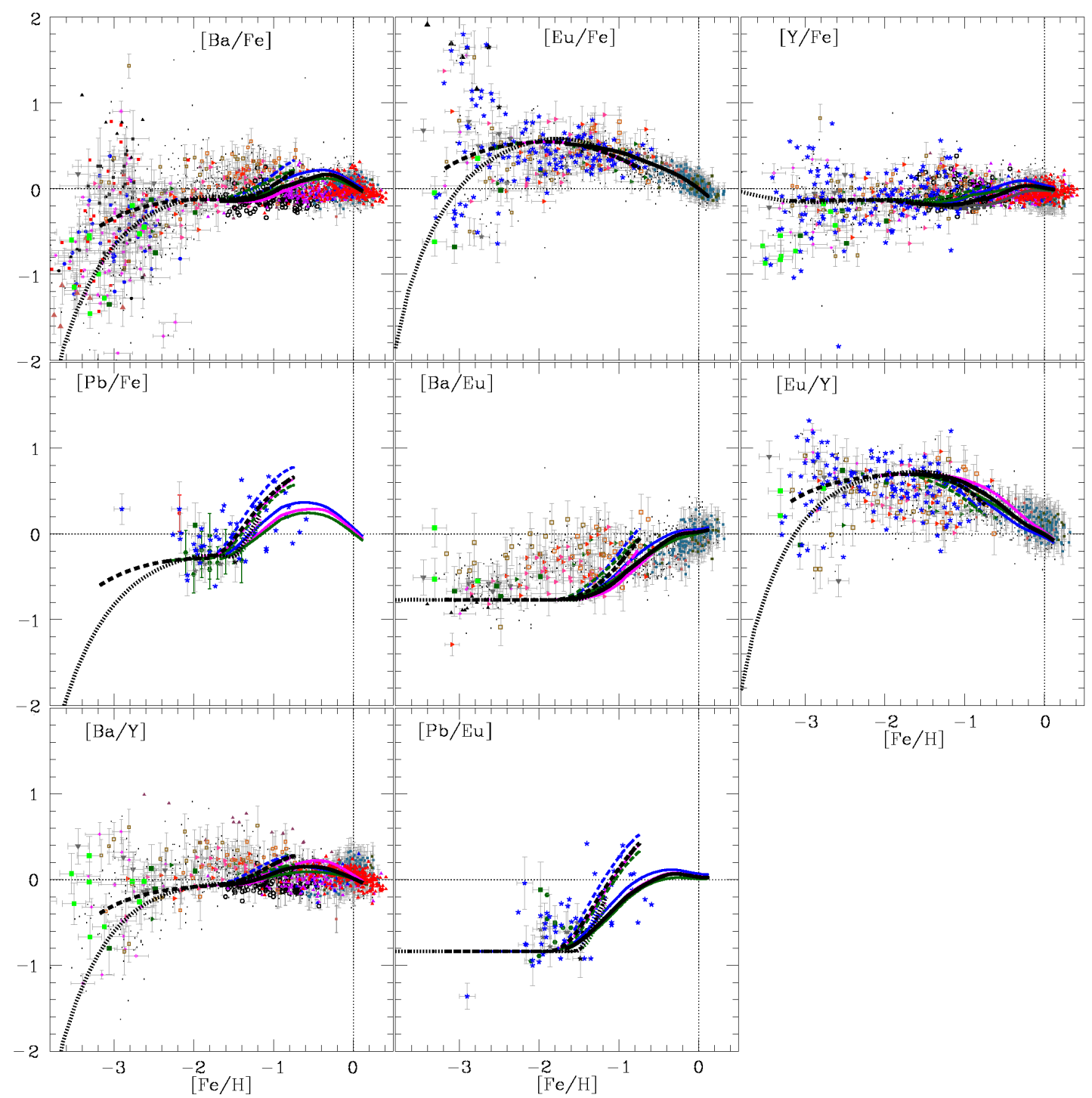

Figure 2. GCE s- and r-process contributions of $[\mathrm{Ba} / \mathrm{Fe}]$, [Eu/Fe], $[\mathrm{Y} / \mathrm{Fe}]$ and $[\mathrm{Pb} / \mathrm{Fe}]$ and their ratios $[\mathrm{Ba} / \mathrm{Eu}],[\mathrm{Eu} / \mathrm{Y}],[\mathrm{Ba} / \mathrm{Y}]$ and $[\mathrm{Pb} / \mathrm{Eu}]$ versus $[\mathrm{Fe} / \mathrm{H}]$. Spectroscopic observations are taken from the compilations by [20], as well as recent updates by [2, 15, 34, 35, 18, 29, 9, 27, 37, 33, 43, $4]$, which include the well studied sample of r-rich stars with $[\mathrm{Eu} / \mathrm{Fe}] \geq+1$. Stars with $[\mathrm{Ba} / \mathrm{Fe}] \geq$ +0.6 and $[\mathrm{Ba} / \mathrm{Eu}]>0$ are excluded (e.g., possible binaries as Ba stars, C-stars, CEMP-s stars).

stellar mass progenitors for the $r$ process, the GCE predictions of $r$ nuclei show marginal variations for disk metallicities, and for the solar s distribution.

The solar abundances are plausibly reproduced within the solar uncertainties (10\% for Y, $6 \%$ for $\mathrm{Ba}, 5 \%$ for $\mathrm{Eu}$ and $7 \%$ for $\mathrm{Pb} ;[24]$ ) at $[\mathrm{Fe} / \mathrm{H}]=0$. Larger variations in $[\mathrm{El} / \mathrm{Fe}]$ predictions (up to $\sim 0.2$ dex, in agreement with an average uncertainty of spectroscopic observations) are shown by $\mathrm{Ba}, \mathrm{Y}$ and $\mathrm{Pb}$ in the metallicity range between $-1.4<[\mathrm{Fe} / \mathrm{H}]<0.1$. Eu, which receives a dominant $r$-process contribution (94\%), is unaffected by the different tests. 
Acknowledgements. S.B. was supported by a JINA Fellowship (NSF Fund PHY-0822648). Numerical calculations have been sustained by B2FH Association (http://www.b2fh.org/).

\section{References}

[1] Abia, C., et al. 2002, ApJ, 579, 817

[2] Aoki, W., et al. 2013, ApJ, 145, 13

[3] Arlandini, C., et al. 1999, ApJ, 525, 886

[4] Bensby, T., et al. 2014, A\&A, 562, 71

[5] Bisterzo, S., Gallino, R., Straniero, O., Cristallo, S., Käppeler, F. 2010, MNRAS, 404, 1529

[6] Bisterzo, S., et al. 2014, ApJ, 787, 10

[7] Busso, M., et al. 2001, ApJ, 557, 802

[8] Busso, M., et al. 2012, 12th Symposium on Nuclei in the Cosmos, PoS, 20

[9] Cohen, J., et al. 2013, ApJ, 778, 56

[10] Cristallo, S., et al. 2011, ApJS, 197, 17

[11] Denissenkov, P. A., Tout, C. A. 2003, MNRAS, 340, 722

[12] Gallino, R., Arlandini, C., Busso, M., et al. 1998, ApJ, 497, 388

[13] Goriely, S., Mowlavi, N. 2000, A\&A, 362, 599

[14] Guandalini, R., Cristallo, S. 2013, A\&A, 555, 120

[15] Hansen, C. J., et al. 2012, A\&A, 545, 31

[16] Herwig, F., Blöcker, T., Schönberner, D., El Eid, M. 1997, A\&A, 324, L81

[17] Herwig, F. 2005, ARA\&A, 43, 435

[18] Ishigaki, M. N., et al. 2013, ApJ, 771, 67

[19] Karakas, A. I., Lattanzio, J. C. 2014, PASA, in press, arXiv:1405.0062

[20] Käppeler, F., Gallino, R., Bisterzo, S., Aoki, W. 2011, Rev. Mod. Phys., 83, 157

[21] Komiya, Y., Yamada, S., Suda, T., Fujimoto, M. Y. 2014, ApJ, 783, 132

[22] Langer, N., Heger, A., Wellstein, S., Herwig, F. 1999, A\&A, 346, L37

[23] Lederer, M. T., Aringer, B. 2009, A\&A, 494, 403

[24] Lodders, K., Palme, H., Gail, H.-P. 2009, Landolt-Börnstein, Edited by J.E. Truemper, 4B, 44

[25] Lugaro, M., Karakas, A., Stancliffe, R. J., Rijs, C. 2012, ApJ, 747, 2

[26] Maiorca, E., et al. 2012, ApJ, 747, 53

[27] Mashonkina, L., Christlieb, N. 2014, A\&A, 565, 123

[28] Matteucci, F., Romano, D., Arcones, A., Korobkin, O., Rosswog, S. 2014, MNRAS, 438, 2177

[29] Mishenina, T., et al. 2013, A\&A, 552, 128

[30] Nucci, M. C., Busso, M. 2014, ApJ, 787, 141

[31] Piersanti, L., Cristallo, S., Straniero, O. 2013, ApJ, 774, 98

[32] Pignatari, M., et al. 2010, ApJ, 710, 1557

[33] Placco, V. M., et al. 2014, ApJ, 781, 40

[34] Roederer, I. U., et al. 2010, ApJ, 724, 975

[35] Roederer, I. U., et al. 2014, MNRAS, 445, 2970

[36] Siess, L., Goriely, S., Langer, N. 2004, A\&A, 415, 1089

[37] Siqueira Mello Jr., C., et al. 2014, A\&A, 565, 93

[38] Straniero, O., Gallino, R., Cristallo, S. 2006, Nucl. Phys. A, 777, 311

[39] Travaglio, C., et al. 1999, ApJ, 521, 691

[40] Travaglio, C., Gallino, R., Busso, M., Gratton, R. 2001, ApJ, 549, 346

[41] Travaglio, C., et al. 2004, A\&A, 425, 1029

[42] Trippella, O., Busso, M., Maiorca, E., Käppeler, F., Palmerini, S. 2014, ApJ., 787, 41

[43] Yong, D., et al. 2013, ApJ, 762, 26 